\title{
II trattamento della patologia degenerativa lombare in videoscopia: nostra esperienza
}

G. Bonfiglio ${ }^{1}$, O.De Bartolomeo ${ }^{1}$, R. Zennato ${ }^{1}$, F.Uboldi² ${ }^{2}$, M. De Vincenzi ${ }^{1}$

${ }^{1}$ Istituti clinici di perfezionamento, CTO, Milano; ${ }^{2}$ Medico frequentatore Istituto Ortopedico G. Pini, Milano

ABSTRACT Surgical videoscopic treatment of degenerative back pain: our experience

Lumbar pain is the main symptom in patients with degenerative lumbar pathology. Sources of this symptomatology are lumbar canal stenosis, disc herniation, yellow ligament and facet joints degeneration, foraminal stenosis and vertebral instability. High percentages of these patients are treated with spinal fusion surgery, lumbar decompression and foraminotomy procedures. Such procedures often cause subsequent development of aderential syndromes. We think that videoscopic procedure could be useful for patients with chronic back pain.

\section{Introduzione}

Nella ricerca di soluzioni alternative per il trattamento delle lombalgie croniche, delle sindromi lombalgiche post-chirurgiche che spesso sono associate a un'oggettività clinica priva di alterazioni o deficit periferici (autentici "rompicapo"), quali patologie degenerative lombari, sindrome post-laminectomia, sindromi aderenziali degenerative e post-chirurgiche, sindromi stenotiche, tante volte la videoscopia del canale lombare ha rappresentato, nella nostra esperienza, una "chance" alternativa.

La videoscopia del canale lombare rappresenta una procedura alternativa, ma applicabile in casi ben selezionati. Lo sviluppo di procedure mini-invasive, ripetibili, caratterizzate da pochi giorni di ricovero per il paziente, rappresenta un'esigenza indotta dalla considerazione che anche le patologie del rachide hanno determinato, nel corso degli ultimi anni, un numero sempre maggiore di accessi in pronto soccorso e negli ambulatori ortopedici e fisiatrici. D'altro canto, occorreva anche valutare un secondo fattore, vale a dire la necessità di rivedere procedure chirurgiche, ovvero l'utilizzazione di strumentari e sistemi protesici sempre più complessi a fronte di una minor aggressività chirurgica.

Sempre più frequentemente si registrano complicanze post-chirurgiche dovute a processi aderenziali, conseguenza delle necessarie soluzioni chirurgiche caratterizzate da esposizione ampia del rachide.

Gli ultimi anni sono stati caratterizzati dalla ricerca di soluzioni sempre meno invasive e, soprattutto, meno demolitive dei tessuti e delle strutture rachidee.

\section{Materiali e metodi}

Dal febbraio 2008 al dicembre 2011 sono stati effettuati 175 interventi chirurgici, di cui 114 in pazienti pazienti mai operati e 61 in pazienti sottoposti negli anni a uno o più interventi chirurgici per lombalgia cronica (laminectomie decompressive, ernie discali, artrodesi vertebrali ecc.). Tutti i pazienti sono stati valutati con scale (Oswestry, Barthel, VAS,
Immpact e Btc scale) e sono stati sottoposti a esami strumentali $(\mathrm{Rx}$, TC, RMN, EMG). Tutti i pazienti sono stati controllati a 15, 30 e 90 giorni da parte sia dell'équipe fisiatrica sia di quella ortopedica. Dopo l'intervento è stata sempre prescritta fisioterapia e i programmi partivano dal $3^{\circ}$ giorno post-operatorio

Le indicazioni alla videoscopia del canale lombare sono costituite da: - dolore lombare cronico e degenerazione discale lombare senza deficit periferici e "non responder" al trattamento farmacologico e fisioterapico

- sindrome algica post-laminectomia

- sindrome stenotica del canale lombare degenerativa e postchirurgica.

- protrusioni ed ernie discali con quadro clinico periferico senza deficit.

Le controindicazioni a questo tipo di trattamento sono rappresentate da:

- coagulopatie

- terapia anticoagulante

- infezion

- alto rischio cardiovascolare

- "non compliance"

- disturbi psichiatrici

Risultati

I risultati attualmente vengono ritenuti ancora provvisori poiché il follow-up è considerato breve.

Abbiamo ottenuto mediamente un risultato positivo nel $75 \%$ dei casi, considerando la riduzione del dolore e il miglioramento della possibilità di adesione ai programmi riabilitativi e di rientro alle attività lavorative. Tale percentuale è riferita a un campione di 109 pazienti ripresentatisi a controlli: 68 pazienti hanno effettuato solo i primi due controlli; 11 casi hanno richiesto la necessità di un trattamento chirurgico tradizionale a distanza di 90 giorni per la mancanza di beneficio dalla procedura; i rimanenti 98 pazienti hanno riferito un $\mathrm{mi}-$ glioramento mediamente nel $75 \%$ dei casi. Nessun paziente ha riferito un peggioramento della sintomatologia pre-intervento. Per risultato positivo si intende:

- regressione della sintomatologia algica e degli episodi lombalgici acuti o sub-acuti recidivanti
- inabilità alla posizione prona.
- regressione della "claudicatio" intermittente di grado iniziale non severo

regressione di ipostenie, inizialmente non severe

- rapido ritorno a una vita quotidiana e lavorativa normale

- ripresa di attività sportiva

- ripristino e recupero della funzionalità del rachide, compatibilmente con i segmenti stabilizzati nei casi sottoposti a chirurgia strumentata.

\section{Discussione}

Da quattro anni viene utilizzata una procedura con tecnica miniinvasiva che permette di eseguire lisi aderenziali e cicatriziali in esiti di laminectomie e non, decompressioni del canale vertebrale lombare con azioni selettive sulle aderenze e, talvolta, asportazione di materiale discale di piccole dimensioni.

La procedura si avvale dell'uso di un catetere (video guida) a diametro variabile (Fig. 1) (28-30 $\mathrm{mm}$ ) con due vie, una per l'introduzione di una fibra ottica da 10.000 pixel e la seconda via per l'introduzione di strumenti chirurgici dedicati.

La tecnica viene eseguita sempre in anestesia locale con accesso dallo iatus sacrale (Fig. 2), praticando un'incisone di $3 \mathrm{~mm}$ a paziente prono.

Viene eseguito un lavaggio del canale vertebrale, a perfusione continua, con soluzione fisiologica 120-150 ml monitorando i volumi di infusione "in/out". Infatti all'interno del canale lombare è opportuno non infondere un quantitativo superiore a $200 \mathrm{ml}$ per evitare il rischio di emorragie retiniche, descritte nella letteratura giapponese.

Linfusione viene addizionata con $4 \mathrm{mg}$ di desametasone, 1 fiala di acido ialuronico e rovipacaina $\mathrm{mg} / 10 \mathrm{ml}$ con infusione separata e discontinua.

La lisi e la decompressione all'interno del canale avvengono con l'uso un palloncino a espansione (Fig. 3) e di una pinza dedicata

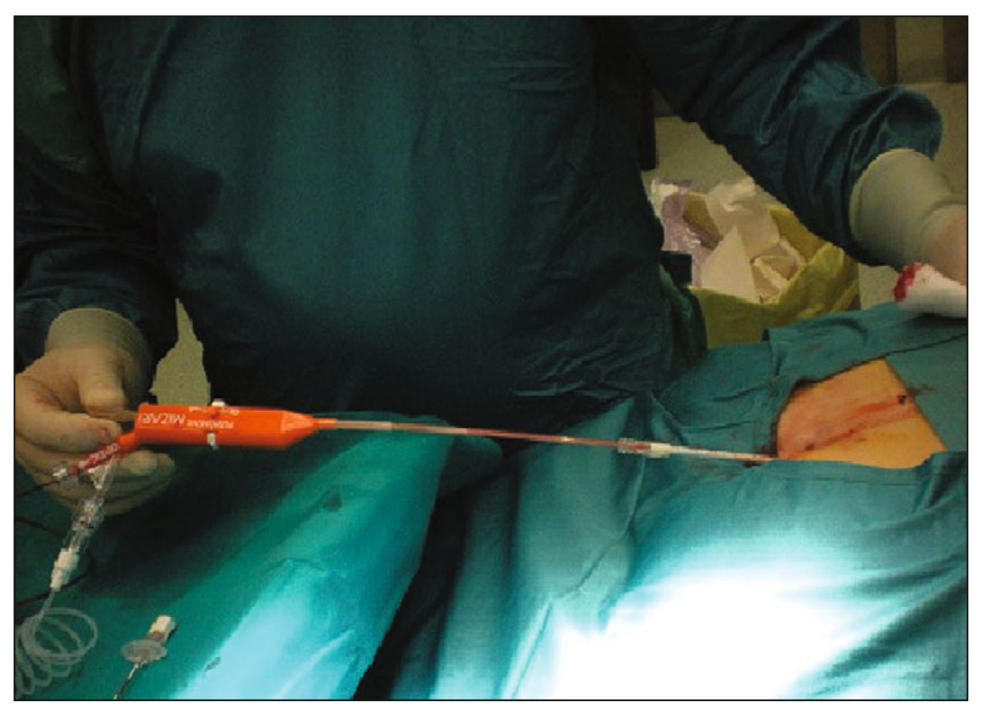

Fig. 1. Posizionamento del paziente, accesso in anestesia locale e introduzione dell'ottica missione periferica o associati se-
001 10.1007/s10261-012-0034-x

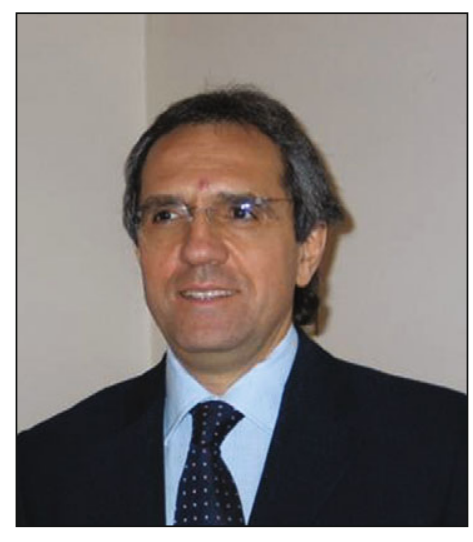
mine il paziente rientra in reparto e può essere dimesso la sera stessa ovvero il giorno successivo.

\section{Conclusioni}

La videoscopia del canale lombare non rappresenta un'alternativa sostitutiva della tradizionale tec-

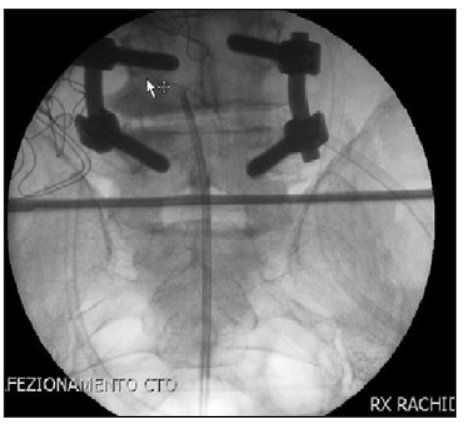

ig. 2. Amplioscopia e valutazione della posizione dell'ottica

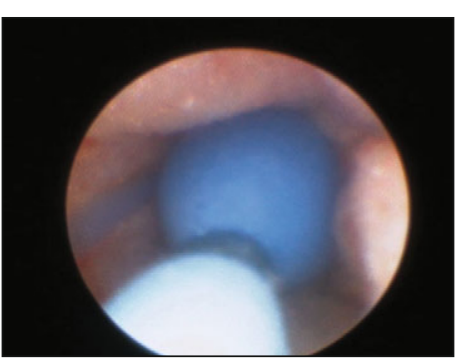

Fig. 3. Momento operativo, lisi aderenziale con palloncino

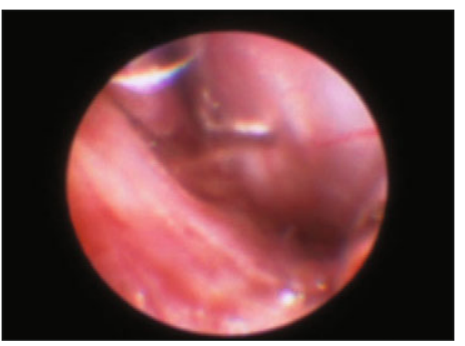

Fig. 4. Lisi aderenziale mediante pinza

nica chirurgica, ma rappresenta un'opzione chirurgica mini-invasiva per tutti quei casi che non rispondono al trattamento farmacologico e fisiochinesiterapico, ma che non presentano una comprogni clinici e strumentali di insta-

(Fig. 4). Normalmente la proceG. Bonfiglio

bilità vertebrali.

Costituisce un'opzione per quei casi, già chirurgicamente trattati ma che sviluppano sindromi aderenziali che non richiedono soluzioni chirurgiche tradizionali e che spesso vengono avviati presso i centri di terapia del dolore. La tecnica videoscopica consente: - rispetto dei tessuti e della mu-

scolatura paravertebrale

- minima e millimetrica demolizione dei tessuti

- esecuzione della procedura chi-

rurgica in anestesia locale

- immediata mobilizzazione e deospedalizzazione del pazien-

- rimozione di tessuto fibroso e aderenziale dal canale vertebrale

con relativa decompressione

- ripetibilità della procedura nel tempo.

Gli sviluppi futuri dovrebbero orientarsi verso una miglior specializzazione dell'ottica e dei "probe" chirurgici, al fine di migliorare le possibilità di visione e lavoro all'interno dello speco. Interessante sarà valutare un maggior numero di pazienti sottoposti a videoscopia in relazione a pazienti non operati, ovvero sottoposti a interventi di stabilizzazione e/o aminectomia.

\section{Letture consigliate}

Dashfield AK, Taylor MB, Cleaver JS, Farrow D (2005) Comparison of caudal steroid epidural with targeted steroid placement during spinal endoscopy for chronic sciatica: a prospective, randomized, double-blind trial. Br J Anaesth 94:514-519

Parkin IG, Harrison GR (1985) The opographical anatomy of the lumbar epidural space. J Anat 141:211-217

Igarashi T, Hirabayashi Y, Seo N et al (2004) Lysis of adhesions and epidural injection of steroid/local anaesthetic during epiduroscopy potential alleviate low back 